\title{
La racine consonantique : évidence dans deux langages secrets en berbère tachelhit
}

Mohamed Lahrouchi et Philippe Ségéral

\section{(2) OpenEdition}

\section{Journals}

Édition électronique

URL : http://journals.openedition.org/rlv/1822

DOI : $10.4000 /$ rlv. 1822

ISSN : 1958-9239

Éditeur

Presses universitaires de Vincennes

\section{Édition imprimée}

Date de publication : 1 décembre 2010

Pagination : 11-30

ISBN : 978-2-84292-264-1

ISSN : 0986-6124

\section{Référence électronique}

Mohamed Lahrouchi et Philippe Ségéral, « La racine consonantique : évidence dans deux langages secrets en berbère tachelhit », Recherches linguistiques de Vincennes [En ligne], 39 | 2010, mis en ligne le 01 décembre 2014, consulté le 02 mai 2019. URL : http://journals.openedition.org/rlv/1822 ; DOI : $10.4000 /$ rlv. 1822 


\title{
Mohamed LAHROUCHI \\ CNRS - Université Paris 8
}

\section{Philippe SÉGÉRAL}

Université Paris 7 - CNRS

\section{LA RACINE CONSONANTIQUE : ÉVIDENCE DANS DEUX LANGAGES SECRETS EN BERBÈRE TACHELHIT}

\begin{abstract}
RÉSUMÉ
Dans cet article, nous argumentons en faveur d'une morphologie à base de racine et gabarit («Root-and-Template»). Nous montrons que ces deux objets sont les unités de base manipulées dans deux langages secrets de femmes, taqjmit et tagnawt. Les locutrices sont capables d'isoler dans les formes-source tachelhit des suites consonantiques de niveau exclusivement radical, et les transforment ensuite par diverses opérations de déguisement. Dans les deux langages secrets, la racine apparaît comme fondamentalement trilitère ; l'épenthèse (en tagnawt) et la reconversion du matériel affixal en consonnes radicales (en taqjmit) permettent aux racines déficitaires bi- et monoconsonantiques héritées des formes tachelhit d'atteindre cette trilitéralité obligatoire. Quant aux gabarits, ils sont fixes, au nombre de deux, incluant des sites morphologiques prédéfinis, qui déterminent le type d'opérations observées dans les formes déguisées (gémination, réduplication, affixation, etc.). Deux contraintes particulières sont, par ailleurs, définies : répétition stricte en taqjmit et satisfaction du gabarit en entier en tagnawt. Ces contraintes permettent d'expliquer certaines différences formelles entre les deux langages, celles liées en particulier à la taille des formes (régulière en tagnawt mais variable en taqjmit) et au comportement de certaines formations quadriconsonantiques (substitution en tagnawt d'un schwa à une voyelle périphérique).
\end{abstract}

\section{MoTS-CLÉS}

Morphophonologie, racine consonantique, gabarits, langages secrets, berbère tachelhit, taqjmit, tagnawt. 
Il est d'usage de décomposer les mots en berbère, comme en sémitique, en des suites ordonnées de consonnes et des mélodies vocaliques. Appelées racines, ces suites consonantiques sont définies dans les études morphologiques, tant traditionnelles que formelles, comme des unités de base, porteuses d'un sens général. Dans les travaux d'obédience générative, à la fin des années 1970, la racine consonantique a été intégrée dans les représentations multilinéaires, où elle est associée à des mélodies vocaliques et à des gabarits spécifiques pour former des mots. Ainsi, pour prendre l'exemple de McCarthy $(1979,1981)$ en arabe classique, la racine $k t b$ 'écrire', associée à la mélodie $a$ et aux gabarits CVCVC, CVCCVC, CVVCVC, forment respectivement les verbes katab, kattab, Paktab et kaatab. Divers travaux théoriques et psycholinguistiques étayent cette hypothèse, montrant que la racine consonantique constitue l'unité de base que les locuteurs stockent dans leur lexique et manipulent dans les opérations morphologiques (Prunet et al., 2000; Davis \& Zawaydeh, 2001). Une revue critique de ces travaux et d'autres est fournie dans Prunet (2006).

Des études récentes, principalement inspirées de la Théorie de l'Optimalité, remettent en cause le rôle de la racine consonantique dans la dérivation, et proposent de former les mots à partir d'autres mots existants (voir, entre autres, Bat El, 1994, 2003; Ratcliffe, 1997; Ussishkin, 1999)1. Les principaux arguments avancés contre ce morphème exclusivement consonantique soulignent son caractère abstrait et son incapacité à rendre compte des phénomènes de transferts mélodiques observés dans certaines formations, tels les verbes dénominaux en hébreu qui héritent aussi bien des propriétés vocaliques que des groupes consonantiques des noms de base (des exemples de type tik 'dossier, classeur' vs tijek 'classer', flirt 'flirt' vs flirtet 'flirter' sont discutés dans Ussishkin, 1999). Des arguments similaires sont fournis dans Dell \& Elmedlaoui (2002 : 55) à propos du berbère.

Dans cet article, nous montrons, à la lumière des données de deux langages secrets pratiqués par des femmes en berbère tachelhit, que les locutrices ont accès à des niveaux de représentation abstraits de leur langue: elles sont capables d'extraire du mot une suite exclusivement consonantique, correspondant à la racine, avant de lui appliquer diverses opérations de déguisement. Nous expliquons ces opérations et les sites sur lesquels elles se produisent comme le résultat direct de l'association d'une racine consonantique, d'une mélodie vocalique et autres éléments affixaux à un gabarit construit, au sens de celui proposé par Guerssel \& Lowenstamm (1990) et Lowenstamm (2003) dans le cas de l'arabe classique, incluant des sites morphologiques spécifiques.

1. Des études alternatives dans le cadre de la Théorie de l'Optimalité incluent la racine consonantique: voir notamment Tucker (dans ce même volume) et Kramer (2006). 
Les variétés de langages secrets étudiées ici sont désignées sous les termes de taqjmit et tagnawt. Le premier terme est probablement lié à l'arabe alSajamia 'langue étrangère, incompréhensible'; le second est la forme féminine de agnaw 'muet'. Les données proviennent, pour le taqjmit, de Lahrouchi \& Ségéral (2009) et, pour le tagnawt, de Douchaïna $(1996,1998)^{2}$.

L'article s'articule en quatre sections. Dans la première, nous examinons les formations triconsonantiques. L'analyse permet de définir les deux gabarits sur lesquels les formations en taqjmit et en tagnawt sont construites et les contraintes qui constituent leurs «règles du jeu» et précise, ensuite, la manière dont la séquence triconsonantique radicale est manipulée. Nous présentons, dans cette même section, quelques éléments théoriques qui permettent de saisir le contenu de l'analyse.

La deuxième section est consacrée à l'étude des formations bi- et monoconsonantiques. Les opérations d'épenthèse à l'œuvre en tagnawt et la reconversion du matériel affixal en consonnes radicales en taqjmit permettent de confirmer le caractère fondamentalement triconsonantique des formes déguisées. Quelques approches alternatives aux faits examinés sont discutées à la fin de cette même section, en particulier l'hypothèse de la circonscription prosodique formulée dans McCarthy \& Prince (1990, 1994). Nous montrons que cette hypothèse n'est pas en mesure de rendre compte des faits observés en taqjmit et en tagnawt.

La troisième section traite des formations quadriconsonantiques. Deux stratégies sont examinées : l'une, présente en taqjmit comme en tagnawt, consiste en l'élimination d'une consonne des racines héritées des formes tachelhit, en conformité avec la condition de trilitéralité; l'autre permet le maintien de toutes les consonnes radicales, mais assortie, en tagnawt, de la substitution d'un schwa [ə] à une voyelle pleine $a$. Cette substitution est interprétée comme un argument en faveur de la représentation en berbère tachelhit des voyelles périphériques comme étant des voyelles phonologiquement longues, associées à deux positions $\mathrm{V}$.

Nous récapitulons nos conclusions dans la section 4 .

\section{Les formations triconsonantiques}

Le tableau en (1) regroupe des formes triconsonantiques tachelhit déguisées en tagnawt (1a) et en taqjmit (1b):

2. Dans le cas du taqjmit, les gloses des formes verbales à l'aoriste en tachelhit sont données à l'impératif. Pour le tagnawt, nous conservons l'infinitif utilisé par Douchaïna (1996, 1998). 


\begin{tabular}{|c|c|c|c|}
\hline \multirow[t]{8}{*}{ a. } & tachelhit & tagnawt & \\
\hline & skr & ajssakrwakr & 'faire' \\
\hline & sawl & ajssawlwawl & 'parler' \\
\hline & i-ksud ${ }^{\mathrm{S}}$ & ajkkasd $^{\text {i }}$ wasd $^{\text {\& }}$ & 'il a peur' \\
\hline & t-afrux-t & ajffarxwarx & 'fille' \\
\hline & $\mathrm{t}$-aknari-t & ajkkanrwanr & 'figues de Barbarie' \\
\hline & $n-s^{i} b r$ & ajss ${ }^{\complement}$ abrwabr & 'nous endurons' \\
\hline & 1-ћml & ajћћamlwaml & 'charge' \\
\hline b. & tachelhit & taqjmit & \\
\hline & frh & tiffarhjurh & 'sois heureux!' \\
\hline & $\mathrm{krf}$ & tikkarfjurf & 'attache!' \\
\hline & $\mathrm{i}-\mathrm{ksud}^{\S}$ & tikkasd $^{\S}$ jusd $^{\S}$ & 'il a peur' \\
\hline & t-amrar-t & timmarrjurr & 'femme' \\
\hline & $t$-afrux-t & tiffarxjurx & 'fille' \\
\hline & 1-axbar & tixxabrjubr & 'informations' \\
\hline & 1-ћsn & tiћћasnjusn & 'nom propre' \\
\hline & m-bark & tibbarkjurk & 'nom propre' \\
\hline
\end{tabular}

Le matériel affixal des formes tachelhit est systématiquement écarté dans les formes déguisées taqjmit et tagnawt: c'est le cas de 1 - et m-, respectivement article défini et morphème participial de l'arabe. C'est aussi le cas du $i$ - de $i-k s u d^{\S}$, marqueur de $3^{\text {e }}$ personne, singulier, masculin, ainsi que du t préfixé et suffixé, marqueur du féminin. On notera que dans tikkasd ${ }^{\S} j u s d^{\S}, i-n^{\prime}$ 'est pas hérité de la forme tachelhit, non plus que le $t$ - initial: toutes les formes taqjmit commencent par $t i$-.

Quant au matériel vocalique des formes tachelhit, il n'est conservé ni en taqjmit ni en tagnawt. Toutes les formes déguisées affichent un vocalisme invariable, indépendant de celui des formes tachelhit:
a. taqjmit $\mathrm{i}-\mathrm{a}-\mathrm{u}^{3}$
b. tagnawt a

3. On remarquera que la vocalisation des formes déguisées en taqjmit, $i$ - $a$ - $u$, suit le «chemin apophonique» mis en évidence pour l'arabe classique par Guerssel \& Lowenstamm (1990, 1996), puis étendu à d'autres langues sémitiques (Ségéral, 1996, 2000), au berbère (Bendjaballah, 1999) et à des langues indo-européennes (allemand par exemple, voir Scheer \& Ségéral, 1998). Le chemin apophonique est exploité en taqjmit sans aucune motivation grammaticale, à la façon dont il l'est dans les formations onomatopéiques et expressives à

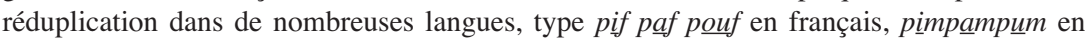
allemand ou espagnol (Lahrouchi \& Ségéral, 2009 : 306). 
En définitive, seules trois consonnes de la forme tachelhit sont conservées dans les formes déguisées: il s'agit des trois consonnes constitutives de la racine. À soi seule, cette observation suffirait à prouver l'existence de la racine et la capacité des locutrices à l'isoler sans erreur. Mais ces langages secrets fournissent d'autres arguments encore dans ce sens, que l'analyse qui suit se propose de faire apparaitre.

Au-delà de la conservation des seules consonnes radicales de la source (désormais $\mathrm{R}_{1}, \mathrm{R}_{2}, \mathrm{R}_{3}$ ), on observe dans les formes déguisées les opérations suivantes :

a. préfixation

i. ti- en taqjmit

ii. aj- en tagnawt

b. gémination de $\mathrm{R}_{1}$

c. insertion de a après $R_{1}$ géminée

d. infixation après $R_{3}$

i. -ju- en taqjmit

ii. -wa- en tagnawt

e. réduplication à droite de $\mathrm{R}_{2}$ et $\mathrm{R}_{3}$

Toutes les formes déguisées issues de trilitères se ramènent donc aux deux schèmes suivants:
a. taqjmit ti
$\mathrm{R}_{1} \mathrm{R}_{1} \quad \mathrm{a}$
$\mathrm{R}_{2} \quad \mathrm{R}_{3} \quad \mathrm{ju}$
$\begin{array}{lll}\mathrm{R}_{2} & \mathrm{R}_{3}\end{array}$
b. tagnawt aj
$\mathrm{R}_{1} \mathrm{R}_{1} \quad \mathrm{a}$
$\begin{array}{lllll}\mathrm{R}_{2} & \mathrm{R}_{3} & \text { wa } & \mathrm{R}_{2} & \mathrm{R}_{3}\end{array}$

Dans les deux cas, on observe que le matériel consonantique de la racine est systématiquement présent deux fois: $\mathrm{R}_{1}$ par gémination, $\mathrm{R}_{2}$ et $\mathrm{R}_{3}$ par réduplication.

La question principale qui se pose ensuite concerne la forme que revêt la répétition des trois consonnes de la racine: pourquoi $R_{1}$ est-elle systématiquement géminée tandis que $\mathrm{R}_{2}$ et $\mathrm{R}_{3}$ sont rédupliquées à droite ? Et comment expliquer la distribution du matériel affixal et vocalique?

Notre hypothèse est que ces opérations de répétition et d'affixation résultent de l'usage de gabarits fixes et structurés, au sens de Guerssel \& Lowenstamm (1990) et Lowenstamm (2003), c'est-à-dire incluant des sites morphologiques, sièges d'opérations particulières. Dans les deux gabarits que nous proposons ci-après, nous attribuons la gémination systématique de 
$\mathrm{R}_{1}$, en tagnawt comme en taqjmit, à la présence d'une syllabe dérivationnelle interne ${ }^{4}$. D'autres sites définis hébergent les affixes listés en (3a, d).

Mais avant de présenter ces gabarits, deux points d'ordre théorique doivent être précisés.

Les gabarits consistent en une suite d'unités $\mathrm{CV}$, en référence au modèle syllabique CVCV (Lowenstamm, 1996). Situé dans le cadre général de la Phonologie du Gouvernement (Kaye et al., 1990), ce modèle stipule que le niveau squelettal des représentations phonologiques consiste en une suite monotone de positions $\mathrm{C}$ et de positions $\mathrm{V}$. Les différents types de syllabes observés en surface résultent des relations de gouvernement que ces positions entretiennent entre elles.

Quant aux segments vocaliques ${ }^{5}$, ils seront, au niveau phonologique, représentés comme associés à deux positions $\mathrm{V}$, bien que les voyelles concernées se réalisent brèves en surface. Cette représentation renvoie à l'hypothèse de la longueur phonologique des voyelles périphériques, initialement proposée par Lowenstamm (1991) dans le cas de l'éthio-sémitique et reprise par Bendjaballah (1999, 2001, 2005) dans le cas du berbère kabyle. Nous reprenons ici cette conception, le tagnawt fournissant de nets arguments supplémentaires en sa faveur ( $c f$. Lahrouchi \& Ségéral, 2010), comme on le verra en particulier dans la section 3 .

Le gabarit que nous proposons pour le tagnawt apparaît en (5) cidessous. La syllabe dérivationnelle interne est soulignée, les séquences encadrées indiquent les parties affixales.

tagnawt

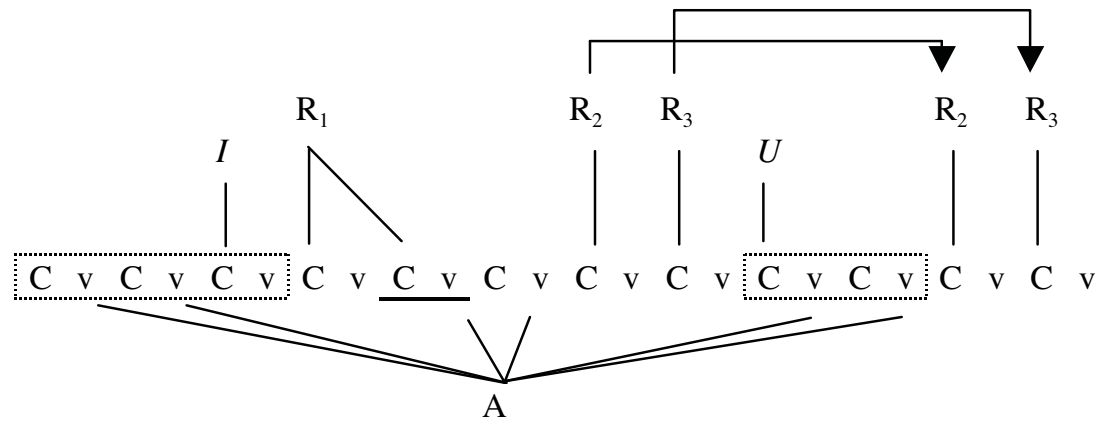

4. On notera que l'on retrouve ici une structure gabaritique tout à fait similaire à celle mise en évidence par Guerssel \& Lowenstamm (1990) pour l'arabe classique: CV [CV $\left.{ }_{\mathrm{DS}}\right]$ $\mathrm{CV} \mathrm{CV}$, où $\left[\mathrm{CV}_{\mathrm{DS}}\right]$ est une syllabe dévolue aux opérations morphologiques internes.

5. Nous représentons les voyelles conformément à la théorie des Éléments (Kaye et al., 1985). Les éléments I et U peuvent être associés à des positions V ou à des positions C. Dans le premier cas, ils sont réalisés comme des voyelles $i$ et $u$, dans le second cas ils apparaissent comme des glides $j$ et $w$. 
Comme il a été dit, les consonnes de la racine sont répétées deux fois: l'une par gémination $\left(\mathrm{R}_{1}\right)$, les deux autres $\left(\mathrm{R}_{2}\right.$ et $\left.\mathrm{R}_{3}\right)$ par réduplication à droite. Le reste des positions dans le gabarit accueille le matériel affixal et vocalique.

On pourrait objecter ici que la taille et la structure du gabarit proposé en (5) pour le tagnawt sont justifiées seulement par l'espace nécessaire à l'accueil du matériel segmental des formes triconsonantiques déguisées.

Mais, en vérité, ce gabarit est unique: ce ne sont pas seulement les formes qui héritent de trois consonnes radicales de la source tachelhit, qui sont dérivées sur le gabarit (5) mais aussi bien celles qui sont issues de biconsonantiques et de monocosonantiques. Considérons en $(6 \mathrm{a}, \mathrm{b}$ et $\mathrm{c})$ un exemple de chacune de ces possibilités:

$\begin{array}{llll} & \text { tachelhit } & \text { tagnawt } \\ \text { a. tri- } & \mathrm{skr} & \text { ajssakrwakr } & \text { 'faire' } \\ \text { b. bi- } & \text { anf } & \text { ajttanfwanf } & \text { 'éviter' } \\ \text { c. mono- } & \mathrm{kk} & \text { ajkkatwatti } & \text { 'traverser' }\end{array}$

Les trois formes déguisées ajkkatwatti, ajttanfwanf et ajssakrwakr occupent un espace phonologique égal, comme cela apparaît en (7) cidessous:
a.
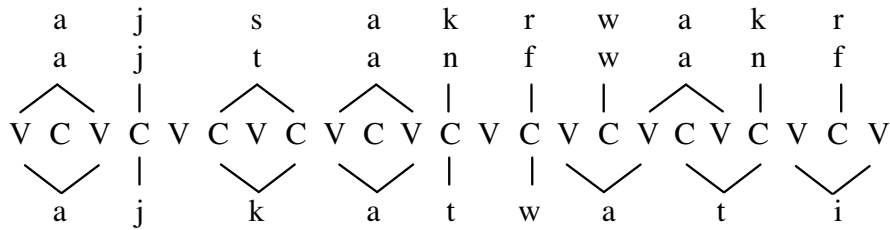

Divers processus permettent aux formations bi- et monoconsonantiques d'atteindre en tagnawt à cette même quantité segmentale, en particulier des processus d'épenthèse consonantique. Ce sera l'objet de la section suivante d'étudier et de préciser ces processus. Pour l'instant, le point important est le volume constant qu'occupent toutes les formes en tagnawt. En termes gabaritiques, cela signifie que le gabarit, unique, est toujours entièrement rempli. L'obligation de satisfaire pleinement le gabarit est une des règles du jeu en tagnawt.

La situation est toute autre en taqjmit. Comparons, comme on vient de le faire pour le tagnawt, trois formes déguisées en taqjmit issues de tri-, bi- et monoconsonantique en tachelhit: 
(8)

\begin{tabular}{llll} 
& \multicolumn{5}{c}{ tachelhit } & taqjmit \\
a. tri- & $\mathrm{krf}$ & tikkarfjurf & 'attache!' \\
b. bi- & gn & tigganjuni & 'dors!' \\
c. mono- & $\mathrm{g}$ & tiggawiwi & 'sois!'
\end{tabular}

La différence saute aux yeux. L'espace occupé par les formes déguisées change suivant le nombre de consonnes radicales reçues des formes tachelhit, comme on le voit en (9):

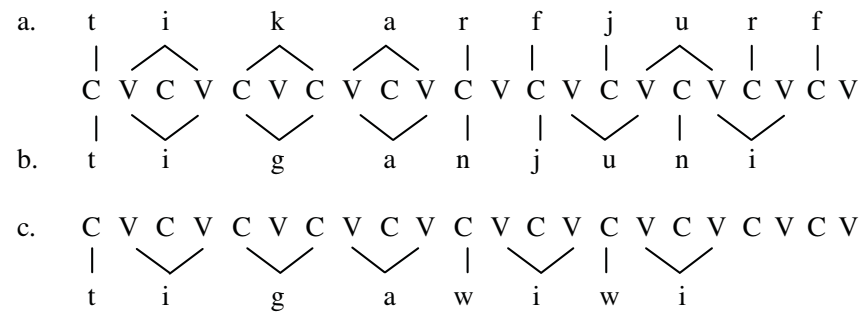

En première analyse, cette différence de taille pourrait s'interpréter comme la conséquence de l'absence d'un gabarit unique en taqjmit; chaque forme déguisée utiliserait un gabarit adapté au nombre de radicales héritées de la forme source.

Mais une observation, cruciale, interdit de voir les choses sous cet angle. En effet les formes taqjmit de (8) aussi bien que les formes tagnawt de (6) manipulent trois objets consonantiques de statut radical, et cela quel que soit le nombre de consonnes radicales héritées du tachelhit. En d'autres termes, la racine en tagnawt ou en taqjmit est triconsonantique ${ }^{6}$, que la racine héritée du tachelhit soit tri-, bi- ou monoconsonantique. Ceci apparaît en (10), où sont repris les exemples de (6) et de (8):

\begin{tabular}{|c|c|c|c|c|c|c|}
\hline \multirow{5}{*}{ a. tagnawt } & \multicolumn{3}{|c|}{ tachelhit } & \multicolumn{3}{|c|}{ formes déguisées } \\
\hline & forme & & racine & forme & & racine \\
\hline & $\mathrm{skr}$ & skr & tri- & ajssakrwakr & skr & \\
\hline & anf & $\mathrm{nf}$ & bi- & ajttanfwanf & $\operatorname{tnf}$ & \\
\hline & $\mathrm{kk}$ & $\mathrm{k}$ & mono- & ajkkatwatti & $\mathrm{ktj}$ & \\
\hline
\end{tabular}

6. À la seule exception de certaines racines quadriconsonantiques, qui demeurent telles dans les formes déguisées. Nous verrons dans la section 3 qu'on observe dans ce cas des opérations particulières d'ajustement du matériel radical au gabarit. Loin d'être des contre-exemples potentiels à l'uniformité des gabarits que nous proposons, ces formes quadriconsonantiques constituent au contraire un argument fort en leur faveur. 
$\begin{array}{lllllll}\text { b. taqjmit } & \text { krf } & \text { krf } & \text { tri- } & \text { tikkarfjurf } & \text { krf } & \text { tri- } \\ & \text { gn } & \text { gn } & \text { bi- } & \text { tigganjuni } & \text { ngj } & \text { tri- } \\ & \text { g } & \text { g } & \text { mono- } & \text { tiggawiwi } & \text { gwj } & \text { tri- }\end{array}$

La différence de volume occupé par les formes du taqjmit ne découle donc pas du nombre de consonnes radicales présentes dans la forme en tachelhit.

En réalité, cette différence provient de l'absence en taqjmit de la contrainte de satisfaction complète du gabarit que nous avons constatée pour le tagnawt. L'absence d'une semblable contrainte découle directement des processus autorisés pour parvenir en taqjmit à la trilitéralité requise. Ce point deviendra évident dans la section suivante.

Il n'y a donc pas de raison de supposer l'existence de plusieurs gabarits en taqjmit. Nous postulons de ce fait un gabarit unique en taqjmit comme en tagnawt.

Quant à la forme exacte de ce gabarit unique, nous ne pouvons pas la justifier pleinement dans le cadre de cet article: pour cela, nous renvoyons le lecteur à Lahrouchi \& Ségéral (2009). Nous nous contenterons de reprendre ici les conclusions de l'article cité. À la différence du tagnawt qui répète seulement la racine, le taqjmit répète la racine mais également un gabarit de base. $\mathrm{Ce}$ gabarit de base correspond à celui mis en évidence par Guerssel \& Lowenstamm (1990) pour l'arabe classique (CV + CV [CV] CV CV) et généralisé à d'autres langues afro-asiatiques, berbères en particulier (Bendjaballah 1999, 2001; Lahrouchi, 2008). Sa répétition génère, en tenant compte de l'hypothèse de la longueur phonologique des voyelles périphériques mentionnée plus haut, le gabarit suivant (11):

\section{(11) taqjmit}

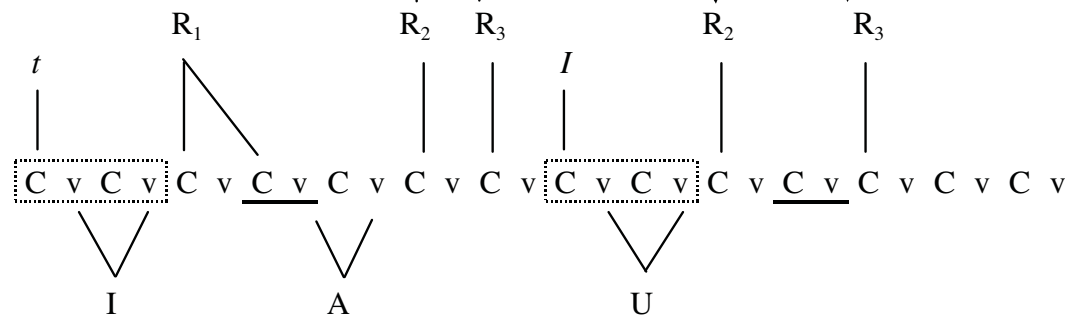

Conformément aux contraintes que nous venons de discuter, le gabarit reste incomplet, les deux dernières syllabes étant non identifiées ${ }^{7}$. L'existence

7. La copie à droite de la syllabe dérivationnelle $[\mathrm{CV}]$ reste inactive : pour des raisons d'architecture morpho-syntaxique, une seule syllabe de ce type peut être activée dans une forme dérivée. Or la première syllabe dérivationnelle est toujours activée en taqjmit. 
de cette partie libre du gabarit trouvera une confirmation dans le comportement de certaines formations en taqjmit issues de mots quadriconsonantiques en tachelhit que nous aborderons dans la section 3 .

Dans la section suivante, nous examinons les formations issues de bi- et monoconsonantiques tachelhit. Celles-ci permettront de préciser les processus au terme desquels le caractère uniformément triconsonantique des racines du tagnawt et du taqjmit est atteint.

\section{Les formations bi- et monoconsonantiques}

Dans le cas des bi- et monoconsonantiques, la trilitéralité requise dans les racines est assurée par l'épenthèse en tagnawt et la reconversion en consonnes radicales du matériel affixal en taqjmit. Nous examinons successivement les deux stratégies adoptées.

\subsection{L'épenthèse (tagnawt)}

En tagnawt, deux éléments épenthétiques, $t$ et $I$, fournissent le matériel radical manquant, comme illustré en (12):

\begin{tabular}{|c|c|c|c|c|}
\hline \multirow[b]{2}{*}{ a. bi- } & tachelhit & tagnawt & $\sqrt{\operatorname{tgn} w}$ & \\
\hline & $\operatorname{ad}^{\oint} n$ & $\operatorname{ajttad}^{\complement} n w a d^{i} n$ & $\operatorname{td}^{\Upsilon} \mathrm{n}$ & 'avoir mal' \\
\hline \multirow{11}{*}{ b. mono- } & anf & ajttanfwanf & $\operatorname{tnf}$ & ‘éviter’ \\
\hline & $a m z^{\complement}$ & $\operatorname{ajttam}^{\complement} w a m z^{\complement}$ & $\operatorname{tmz}$ & 'attraper' \\
\hline & i-fl & ajffalwalli & flI & 'il a laissé' \\
\hline & sala & ajssalwalli & slI & 'être occupé' \\
\hline & kl & ajkkalwalli & klI & 'passer la journée' \\
\hline & ili & ajttalwalli & tlI & 'être' \\
\hline & $i-z z^{\complement} a$ & ajzz ${ }^{\complement}$ atwatti & $z^{i} \mathbf{t I}$ & 'il a poursuivi' \\
\hline & $\iint$ & 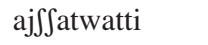 & $\int t I$ & 'manger' \\
\hline & $\mathrm{kk}$ & ajkkatwatti & ktI & 'traverser' \\
\hline & $t-33^{i-t}$ & aj33atwatti & $3 \mathbf{t I}$ & 'tu es guéri' \\
\hline & is & ajssatwatti & stI & interr. 'est-ce que ?' \\
\hline
\end{tabular}

Dans les formes issues de racines biconsonantiques (12a), on observe une épenthèse de $t$ en $\mathrm{R}_{1}$, de $I$ en $\mathrm{R}_{3}$. Dans les formes monoconsonantiques (12b), épenthèse de $t$ et de $I, t$ en $\mathrm{R}_{1}$ ou $\mathrm{R}_{2}, I$ en $\mathrm{R}_{3}{ }^{8}$.

8. Les paramètres en fonction desquels les locutrices déterminent dans une forme tachelhit bi- ou monoconsonantique donnée quelle est la radicale (ou les radicales) manquante(s) sur les trois requises nous restent peu claires. 
Dans le cas où c'est la première radicale qui est considérée comme manquante, le $t$ épenthétique qui apparaît gémine comme attendu dans cette position.

Lorsque c'est la troisième radicale qui est considérée comme absente dans la forme tachelhit, l'Élément épenthétique $I$ qui apparaît alors provoque des ajustements plus complexes. Notons tout d'abord que sa forme rédupliquée, à la fin de la forme, est toujours vocalisée: il apparaît toujours sous la forme d'un $i$ final. En tachelhit, en effet, un $I$ associé à une position $\mathrm{C}$ précédée et suivie d'un noyau vide est régulièrement vocalisé: par ex. 'monter' $\gamma^{w} l i$ (aoriste) vs $a q q^{w} l a j$ (inaccompli), 'passer' zri (aoriste) vs zraj (inaccompli). Ensuite, on observe deux phénomènes conjoints:

a. le $I$ épenthétique n'apparaît jamais à l'endroit attendu pour $\mathrm{R}_{3}$, c'està-dire immédiatement avant le -wa- infixal (ajttanfwanf, mais *ajffalliwalli, *ajkkatIwatti). Les formations tagnawt avec I épenthétique ne présentent jamais qu'une seule consonne avant le - wa- infixal et ce sont les seules formes dans ce cas;

b. l'image rédupliquée de $\mathrm{R}_{2}$ - que ce soit une radicale héritée ou un $t$ épenthétique - est systématiquement géminée: ajttalwalli, ajkkatwatti $i$ etc. Là encore, seules les formes à $I$ épenthétique présentent cette caractéristique en tagnawt.

En réalité, l'épenthèse de $I$ en $\mathrm{R}_{3}$ génère dans tous les cas une séquence sous-jacente - $C j w$-, le $w$ de l'infixe - wa-faisant immédiatement suite à $\mathrm{R}_{3}$. Or une telle séquence semble prohibée et la stratégie adoptée consiste en l'élimination du premier des deux glides adjacents ${ }^{9}:{ }^{*} C j w-\rightarrow C w$. Cette élimination a pour conséquence qu'une des positions du gabarit n'est pas identifiée: la gémination de l'image rédupliquée de $\mathrm{R}_{2}$ qu'on observe alors est la stratégie adoptée pour compenser ce manque, le gabarit devant être, rappelons-le, entièrement satisfait en tagnawt.

Les représentations en (13) illustrent l'ensemble des situations qu'on vient de discuter: épenthèse de $t$ en $\mathrm{R}_{1}$ (13a), de $I$ en $\mathrm{R}_{3}(13 \mathrm{~b})$, de $t$ en $\mathrm{R}_{2}$ et de $I$ en $\mathrm{R}_{3}(13 \mathrm{c})$ :

9. La même opération semble justifier l'absence de $u$, marqueur de l'état construit, dans les formes masculines plurielles en berbère: on a par exemple au singulier argaz (état libre) vs urgaz (état construit) 'homme', mais au pluriel on trouve irgazn (état libre et construit), là où on s'attendrait à *wirgazn pour l'état construit. 
(13) a. $\quad \operatorname{ad}^{\S} n \rightarrow \operatorname{ajttad}^{\complement} n w a d^{\complement} n$

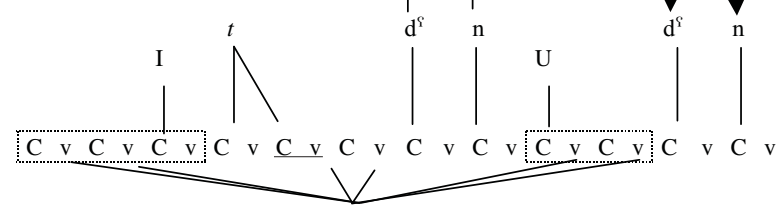

A

b. $\mathrm{kl} \rightarrow$ ajkkalwalli

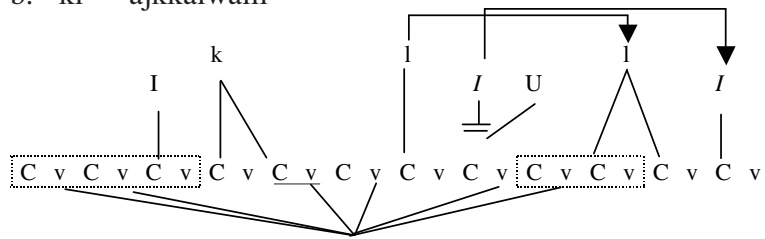

A

c. is $\rightarrow$ ajssatwatti

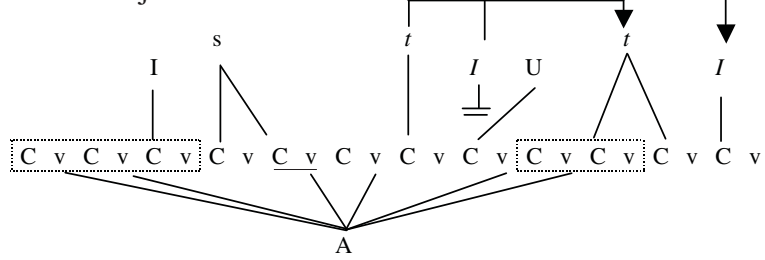

En tagnawt, donc, si la racine héritée n'est pas triconsonantique, le matériel consonantique manquant est obtenu par voie d'épenthèse.

\subsection{La reconversion en consonnes radicales du matériel affixal (taqjmit)}

Contrairement au tagnawt, le taqjmit ignore rigoureusement toute forme d'épenthèse consonantique.

Considérons les exemples ci-dessous :

\begin{tabular}{|c|c|c|c|}
\hline \multirow[t]{5}{*}{ a. bi- } & $\begin{array}{l}\text { tachelhit } \\
\text { gn }\end{array}$ & $\begin{array}{l}\text { taqjmit } \\
\text { tigganjuni }\end{array}$ & 'dors!' \\
\hline & jr & tiyyarjuri & 'lis!' \\
\hline & igr & tiggarjuri & 'champs' \\
\hline & ils & tillasjusi & 'langue' \\
\hline & ৎi\a & 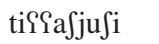 & nom propre \\
\hline \multirow[t]{5}{*}{ b. mono- } & $\mathrm{g}$ & tiggawiwi & 'sois!' \\
\hline & af & tiffawiwi & 'trouve!' \\
\hline & asi & tissawiwi & 'prends!' \\
\hline & ini & tinnawiwi & 'dis!' \\
\hline & immi & timmawiwi & 'ma mère' \\
\hline
\end{tabular}


À première vue, la voyelle $i$ qui apparaît en position finale de toutes ces formes devrait s'analyser comme le résultat de l'épenthèse d'un Élément $I$, comme en tagnawt. Mais si les formations biconsonantiques se prêtent facilement à cette interprétation - le $i$ final remplaçant dans ce cas $\mathrm{R}_{3}$ manquante, les monoconsonantiques posent le problème de l'interprétation du suffixe -wiwi qu'on observe, conjugué dans les mêmes formes à l'absence de -ju-, affixe commun à toutes les autres formes (bi- et triconsonantiques).

En réalité, aucun matériel épenthétique n'est ajouté aux formes bi- et monoconsonantiques en taqjmit. Le - $i$ final des premières et le -wiwi suffixé aux secondes ne sont que le produit d'une réutilisation du matériel affixal déjà présent dans les formes, les Éléments $I$ et $U$, composants mêmes de l'affixe -ju-. Les processus de reconversion de ces Éléments affixaux en matériel radical sont figurés ci-dessous en (15a) pour les biconsonantiques et en (15b) pour les monoconsonantiques:

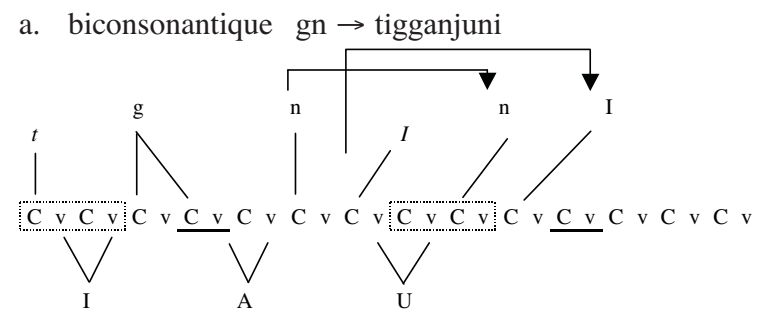

b. monoconsonantique

$\mathrm{g} \rightarrow$ tiggawiwi

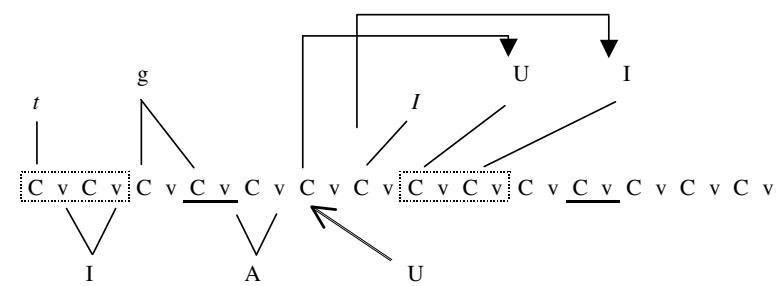

En (15a), $g$ gémine et $n$ réduplique à droite. La position $R_{3}$ étant vide, l'élément affixal $I$ s'y associe et réduplique ensuite à droite, comme n'importe quelle autre radicale. En (15b), la situation est plus complexe: ayant hérité d'une racine monoconsonantique, la forme déguisée mobilise les deux éléments affixaux $I$ et $U$. Ce dernier prend place en $\mathrm{R}_{2}$ et $I$ s'associe à $\mathrm{R}_{3}$. La totalité du matériel est ensuite transformée comme attendu : $g$ gémine, $U$ et $I$ rédupliquent à droite, d'où leur forme de surface: wiwi.

On notera que le gabarit reste incomplet dans les deux représentations. C'est là une différence capitale entre le taqjmit et le tagnawt. En taqjmit, aucun matériel épenthétique n'est jamais ajouté pour remplacer les consonnes radicales manquantes. Seul le matériel affixal déjà présent dans la forme est mobilisé. 
De même, aucun segment associé à une des positions de la racine, qu'il soit d'origine radicale ou affixale, n'est présent plus de deux fois dans la forme déguisée: jamais on n'a en taqjmit réduplication et gémination d'une même consonne. Cette répétition stricte (id est excluant réduplication et gémination) explique l'incomplétude du gabarit dans les formes bi- et monoconsonantiques du taqjmit.

Par-delà la différence dans les stratégies retenues (épenthèse en tagnawt, mobilisation du matériel affixal en taqjmit), ce qui apparaît fondamental c'est bien la contrainte sur la forme des racines: toute racine, en taqjmit comme en tagnawt, doit être triconsonantique.

Cela dit, on observera que toutes les formes déguisées, tri-, bi- et monoconsonantiques, présentent une partie commune, invariable: $\# \mathrm{tiC}_{1} \mathrm{C}_{1} \mathrm{a}-$ en taqjmit et \#ajC $\mathrm{C}_{1} \mathrm{C}_{1} \mathrm{a}$ - en tagnawt. Cette constatation pourrait inviter à ne voir dans les stratégies analysées plus haut que des opérations morphophonologiques secondaires par rapport à l'existence de ces parties invariables. De plus celles-ci seraient susceptibles d'être définies en termes purement prosodiques. De sorte que l'on pourrait être tenté d'analyser les faits du taqjmit et du tagnawt dans le cadre de la théorie de la circonscription prosodique proposée par McCarthy \& Prince $(1990,1994)$. Analysant le cas des pluriels brisés et des diminutifs de l'arabe classique (par ex. sg. zundub 'criquet', pl. zanaadib, dim. zunajdib;

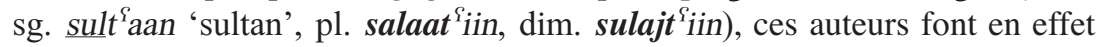
valoir qu'ils offrent une portion prosodique invariable (un pied iambique, en gras dans les exemples), construit à partir d'une portion définie (soulignée) de la forme singulier. Ce modèle rend également compte de l'existence de propriétés communes aux formes de base (sg.) et aux formes dérivées (pl. et dim.) en termes de transfert de longueur vocalique : la voyelle finale des formes du singulier garde sa longueur dans les formes plurielles et diminutives.

Mais tout d'abord, il n'y a aucun transfert de propriété du type discuté dans McCarthy \& Prince observable entre les formes tachelhit et les formes déguisées taqjmit et tagnawt. Ensuite et surtout, dans un tel cadre, on passerait à côté de généralisations importantes que la morphologie à base de racine et gabarit permet de capturer. Les parties variables opposant entre elles les formations issues de tri-, bi- et monoconsonantiques offrent en effet des régularités morphophonologiques qui s'interprètent de manière naturelle dans un modèle incluant racine consonantique et gabarit construit. Ainsi, en taqjmit, c'est le comportement, dans un gabarit construit, des Éléments $I$ et $U$ qui explique les variations des formes bi- et monoconsonantiques par rapport aux formes triconsonantiques. De même, en tagnawt, ce sont les contraintes gabaritiques qui expliquent le recours à l'épenthèse, source des différences entre les formes triconsonantiques et les autres. Nous ne voyons pas comment ces variations peuvent être interprétées logiquement dans l'approche prosodique de McCarthy \& Prince. Enfin et surtout, les formes déguisées se construisent à 
partir de racines triconsonantiques, soit extraites de la source, soit construites au terme de processus divers lorsque la racine tachelhit offre moins des trois consonnes requises, et ce fait majeur ne nous semble pas pouvoir trouver une explication adéquate dans le cadre de la circonscription prosodique.

Les formations quadriconsonantiques, vers lesquelles nous allons nous tourner dans la section suivante, confirment le caractère central de cette contrainte de trilitéralité de la racine.

\section{Les formations quadriconsonantiques}

À côté des formes issues de racines bi- et monoconsonantiques en tachelhit, il existe, aussi bien en taqjmit qu'en tagnawt, des formes construites à partir de quadriconsonantiques en tachelhit.

Deux stratégies s'appliquent à ces formations quadriconsonantiques: soit le nombre de consonnes est ramené à trois par élimination d'une radicale, soit toutes les consonnes sont préservées, mais dans ce cas, diverses opérations d'ajustement interviennent.

Commençons par considérer en (16) quelques exemples en tagnawt:

\begin{tabular}{|c|c|c|c|}
\hline \multirow{3}{*}{ a. } & tachelhit & tagnawt & \\
\hline & asrdun & ajssadnwadn & 'mule' \\
\hline & aglzim & ajggazmwazm & 'pioche' \\
\hline & asngar & ajssagrwagr & ‘maïs’ \\
\hline & g3 dr & ajggazdwazd & 'se lamenter' \\
\hline & wwnzr & ajwwanzwanz & 'saigner du nez' \\
\hline & $\operatorname{amaz}^{\complement} \mathrm{duj}$ & $\operatorname{ajmmaz}^{\complement} \mathrm{dwaz}^{\complement} \mathrm{d}$ & 'lourd' \\
\hline b. & xurb $\int$ & ajxxərbfwab $\int$ & 'griffer' \\
\hline & aknbus & 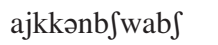 & 'foulard' \\
\hline & 1lustm & ajlləetmwatm & 'ramper' \\
\hline & ggrml & ajggərmlwaml & 'être croustillant' \\
\hline & agnfur & ajggənfrwafr & 'gueule' \\
\hline
\end{tabular}

Les formes en (16a) exemplifient le cas où une radicale est supprimée, ramenant leur nombre à trois. Dans les trois premières formes, c'est $\mathrm{R}_{2}$ qui est éliminée; dans les trois autres, c'est $\mathbf{R}_{4}$. Dans tous les cas, il s'agit d'une consonne sonante ${ }^{10}$. Les opérations attendues s'appliquent ensuite: $\mathrm{R}_{1}$ gémine et les deux autres radicales rédupliquent à droite.

10. Une stratégie similaire s'observe dans les quinquilitères de l'arabe classique qui forment leurs pluriels nominaux par élimination d'une radicale, souvent sonante, en position médiane ou finale: par exemple, safarzal (sg)/safaariz (pl) 'coing', barnaamaz (sg)/ baraamiz (pl) 'programme', Sandaliib (sg)/Sanaadil (pl) 'rossignol' (voir McCarthy, 1979 : 187 ; Moujib Dupont, 1989). 
Les formes en (16b) se comportent différemment: aucune des quatre radicales n'est éliminée, mais l'une d'entre elles, $\mathrm{R}_{2}$, échappe à la répétition. La raison de ce traitement est évidente: le gabarit ne permet tout simplement pas d'accommoder quatre consonnes répétées. La non-répétition d'une consonne lorsqu'on en conserve quatre est ainsi une confirmation nette du gabarit proposé.

Mais dans les formes (16b), on note en outre que le $a$ qui fait suite normalement à $\mathrm{R}_{1}$ géminée est remplacé par un schwa [ə]. Ce dernier fait s'interprète naturellement comme la conséquence de l'association de quatre consonnes radicales à un gabarit fondamentalement conçu pour n'en accueillir que trois. La situation est figurée en (17):

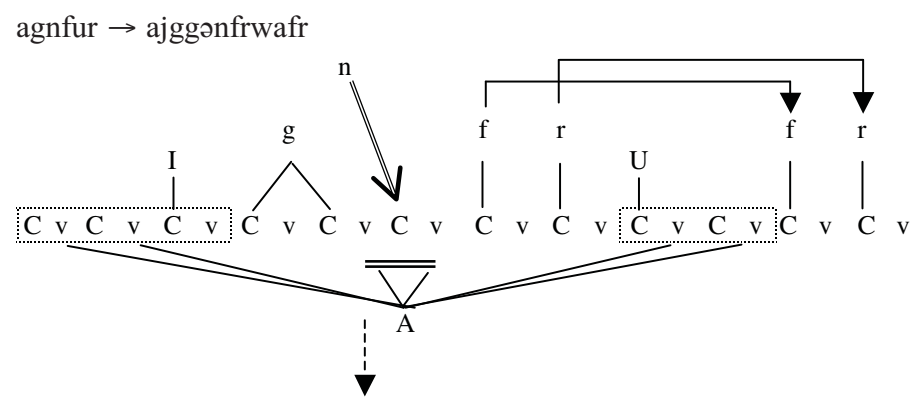

[ə]

Le maintien des quatre consonnes radicales dans la forme tagnawt conduit la deuxième radicale $n$ à s'associer à la position consonantique située à droite de $\mathrm{R}_{1}$ géminée. Ceci a pour conséquence que la voyelle $\mathrm{A}$ n'a plus accès à deux positions vocaliques. Dans le cadre de l'hypothèse de la longueur phonologique des voyelles périphériques ( $c f$. section 1), seul un schwa peut se réaliser sur une position $\mathrm{V}$ unique. En dehors de ce cadre, il ne nous paraît pas possible de rendre compte du fait que toutes les racines quadriconsonantiques qui gardent leurs consonnes en tagnawt, et seulement celles-là, présentent un schwa après $R_{1}$ (pour un argumentaire détaillé sur ce point, voir Lahrouchi \& Ségéral, 2010).

En taqjmit, la manipulation des racines quadriconsonantiques est légèrement différente. Le nombre des consonnes est, comme en tagnawt, soit ramené à trois (18a), soit maintenu (18b). Mais dans ce dernier cas, aucun élément vocalique n'est éliminé, contrairement à ce qui est observé en tagnawt. 
(18)

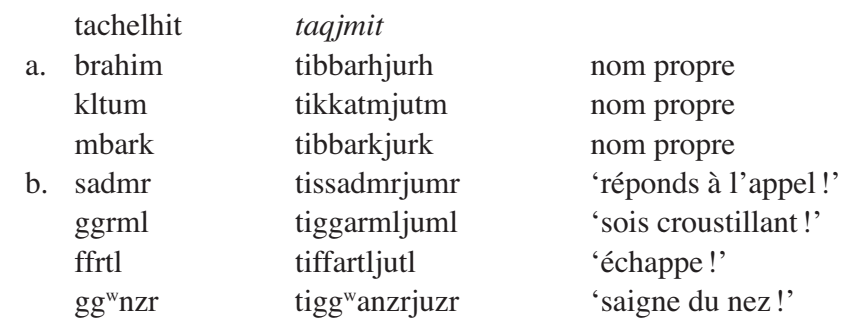

En (18a), une consonne est éliminée, soit $\mathrm{R}_{2}$, soit $\mathrm{R}_{4}{ }^{11}$. En (18b) en revanche, les quatre consonnes radicales sont maintenues et, comme en tagnawt, seules trois d'entre elles sont répétées. Mais contrairement au tagnawt cela n'entraîne aucune opération d'ajustement puisque, comme expliqué dans la section 1, le gabarit du taqjmit offre suffisamment d'espace pour que tout le matériel radical soit associé. Et pour la même raison, on n'assiste pas en taqjmit, contrairement à ce qui arrive en tagnawt dans le même cas, à l'apparition de schwa dans la mélodie: le gabarit n'étant pas saturé, toutes les voyelles disposent des deux positions $\mathrm{V}$ requises pour faire surface.

Nous donnons en (19) une représentation (sadmr $\rightarrow$ tissadmjumr) qui illustre ce qui vient d'être dit:

sadmr $\rightarrow$ tissadmjumr

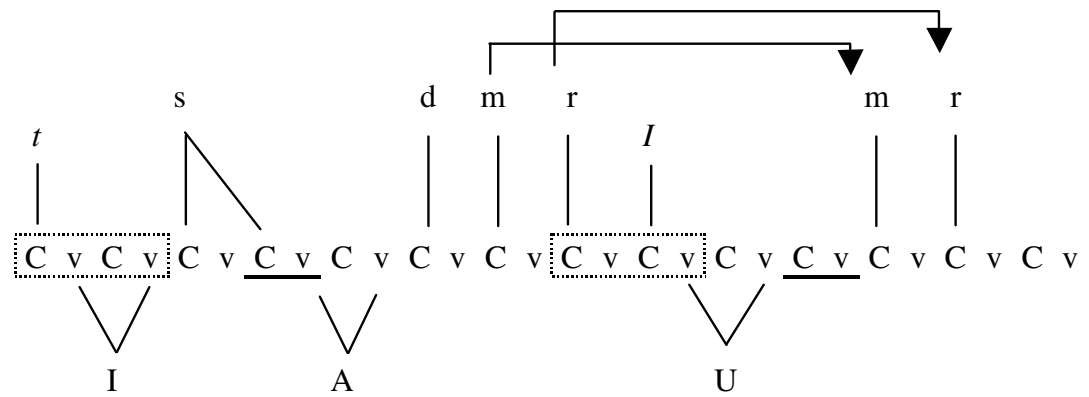

Les formes déguisées issues de quadriconsonantiques tachelhit permettent donc de préciser la contrainte de trilitéralité de la racine en taqjmit et en tagnawt: la racine dans les deux langages secrets doit être au moins triconsonantique.

Au-delà, l'apparition systématique d'un schwa dans la mélodie en tagnawt, mais non en taqjmit, dans le cas où la racine retenue comporte plus de trois consonnes, non seulement confirme la structure des gabarits proposés et

11. Dans le cas de mbark, on peut se demander si la consonne supprimée est interprétée comme un préfixe ou comme une consonne radicale. En arabe, d'où ce nom est issu, $m$ - est un morphème du participe. 
les règles du jeu différentes reconnues pour chacun des deux langages secrets, mais encore constitue un argument fort en faveur de la longueur phonologique des voyelles périphériques proposée en section 1 .

\section{Conclusion}

La racine et le gabarit sont ainsi clairement les deux réalités linguistiques qui sont manipulées dans le cadre des deux langages secrets de femmes que sont le tagnawt et le taqjmit.

Les locutrices, tout d'abord, sont capables d'extraire, sans erreur, le matériel consonantique radical des formes du tachelhit: il n'est jamais conservé quoi que ce soit d'autre de celles-ci dans les formes déguisées. Mais, de surcroît, elles sont capables de construire un morphème radical répondant à une contrainte simple (trilitéralité minimale); les moyens employés diffèrent de l'un à l'autre langage secret, épenthèse en tagnawt et réaffectation des éléments affixaux en taqjmit, et cette différence s'analyse comme résultant de «règles du jeu» légèrement différentes (pleine satisfaction du gabarit en tagnawt, répétition stricte en taqjmit), mais dans tous les cas un morphème radical au moins triconsonantique est construit.

Les mêmes locutrices sont capables d'un autre côté de projeter ce morphème radical ainsi que d'autres éléments affixaux et mélodiques sur des gabarits construits, incluant des sites internes ou affixaux, sièges d'opérations morphologiques particulières.

La forme des deux langages secrets analysés milite donc clairement pour une morphologie «Root-and-Template» où racine consonantique et gabarit sont des morphèmes à part entière.

\section{RÉFÉRENCES}

Bat El, Outi (1994). Stem modification and cluster transfer in Modern Hebrew. Natural Language and Linguistic Theory 12: 571-596.

BAT El, Outi (2003). The fate of the consonantal root and the binyan in Optimality Theory. Recherches Linguistiques de Vincennes 32: 31-60.

Bendjaballah, Sabrina (1999). Trois Figures de la structure interne des gabarits : activité morphologique du niveau squelettal des représentations phonologiques en berbère, somali et bedja. Thèse de Doctorat, Université Paris 7.

Bendjaballah, Sabrina (2001). The «negative preterit» in Kabyle Berber. Folia Linguistica XXXIV/3-4: 185-220.

Bendjaballah, Sabrina (2005). Longueur phonologique des voyelles en kabyle. Études et documents berbères 22 : 47-69.

Davis, Stuart; ZaWAYdeh, Bushra (2001). Arabic hypocoristics and the status of the consonantal root. Linguistic Inquiry 32 : 512-520.

Dell, François; Elmedlaou, Mohamed (2002). Syllables in Tashlhiyt Berber and in Moroccan Arabic. Dordrecht: Kluwer Academic Publishers. 
Douchaïna, Rqia (1996). Tagnawt, un parler secret des femmes berbères de Tiznit (sud-ouest marocain). Thèse de Doctorat, INALCO.

DouchaïnA, Rqia (1998). La morphologie du verbe en tagnawt. Études et documents berbères 15/16: 197-209.

Guerssel, Mohamed; Lowenstamm, Jean (1990). The Derivational Morphology of the Classical Arabic Verbal System. Ms. UQAM \& Université Paris 7.

Guerssel, Mohamed; Lowenstamm, Jean (1996). Ablaut in Classical Arabic measure I active verbal forms. In Lecarme, Schlonsky \& Lowenstamm (eds.), Studies in Afroasiatic Grammar: 123-134. The Hague: Holland Academic Graphics.

IDRISSI, Ali (2000). On Berber plurals. In Lecarme, Lowenstamm \& Shlonsky (eds.), Research in Afroasiatic Grammar: 101-124. Amsterdam: John Benjamins.

KaYe, Jonathan; Lowenstamm, Jean; Vergnaud, Jean-Roger (1985). The internal structure of phonological elements : a theory of charm and government. Phonology Yearbook $2: 305-328$.

Kaye, Jonathan; Lowenstamm, Jean; Vergnaud, Jean-Roger (1990). Constituent structure and government in phonology. Phonology 7/2: 193-231.

Kramer, Ruth (2006). Root and pattern morphology in Coptic: evidence for the root. In Davis, Deal \& Zabbal (eds.), The Proceedings of NELS 36: 399-412. Amherst: GLSA.

LAHroUCHI, Mohamed (2008). A templatic approach to gemination in the imperfective stem of Tashlhiyt Berber. Studies in African Linguistics 37/1: 21-60.

Lahrouchi, Mohamed; SÉGÉral, Philippe (2009). Morphologie gabaritique et apophonie dans un langage secret féminin (taqjmit) en berbère tachelhit. Revue canadienne de linguistique 54: 291-316.

LAHrouchi, Mohamed; SÉGÉrAL, Philippe (2010). Peripheral vowels in Tashlhiyt Berber are phonologically long: evidence from Tagnawt, a secret language used by women. Brill's Annual of Afroasiatic Languages and Linguistics, 2: 202-212.

Lowenstamm, Jean (1991). Vocalic length and centralization in two branches of Semitic (Ethiopic and Arabic). In Kaye (ed.), Semitic Studies in Honor of Wolf Leslau on the Occasion of his Eighty-Fifth Birthday: 949-965. Wiesbaden: Otto Harrassowitz.

Lowenstamm, Jean (1996). CV as the only syllable type. In Durand \& Laks (eds.), Current Trends in Phonology: Models and Methods, vol. 2: 419-441. Salford: European Studies Research Institute, University of Salford.

Lowenstamm, Jean (2003). À propos des gabarits. Recherches Linguistiques de Vincennes $32: 7-30$.

McCarthy, John (1979). Formal Problems in Semitic Phonology and Morphology. Doctoral dissertation, MIT, Cambridge, Massachussets.

McCARTHY, John (1981). A prosodic theory of nonconcatenative morphology. Linguistic Inquiry 12 : 373-418.

McCarthy, John; Prince, Alan (1990). Foot and word in prosodic morphology: The Arabic broken plural. Natural Language and Linguistic Theory 8: 209-283.

McCarthy, John; Prince, Alan (1994). Prosodic morphology. In Goldsmith (ed.), The Handbook of Phonological Theory: 318-366. Cambridge, Massachussets: Blackwell.

MoujiB Dupont, Ilham (1989). La formation des pluriels brisés issus des quinquilitères et des sextilitères en arabe. In Chekayri et Bohas (eds), Languages and Linguistics, vol. 2: Issues in Arabic morphology: Empirical and Theoretical Perspectives: 93145. Dordrecht: Kluwer. 
Prunet, Jean-François (2006). External evidence and the Semitic root. Morphology 16: 4167.

Prunet, Jean-François; Béland, Renée; IdRISSI, Ali (2008). The mental representation of Semitic words. Linguistic Inquiry 31/4 : 609-648.

Ratcliffe, Robert (1997). Prosodic templates in a word-based morphological analysis of Arabic. In Eid \& Ratcliffe, Perspectives on Arabic Linguistics X: 147-171. Amsterdam/Philadelhia: John Benjamins.

Scheer, Tobias; SÉGÉral, Philippe (1998). A generalized theory of ablaut: the case of Modern German strong verbs. Dans Ortmann, Fabri \& Parodi, Models of Inflection: 28-59. Tübingen: Niemeyer.

SÉgéral, Philippe (1996). L'apophonie en ge'ez. In Lecarme, Lowenstamm \& Shlonsky (eds), Studies in Afroasiatic Grammar: 360-391. The Hague: Holland Academic Graphics.

SÉGÉRAL, Philippe (2000). Théorie de l'apophonie et organisation des schèmes en sémitique. In Lecarme, Lowenstamm \& Schlonsky (eds), Research in Afroasiatic Grammar II: 263-299. Amsterdam/Philadelphia: John Benjamins.

Ussishrin, Adam (1999). The inadequacy of the consonantal root: Modern Hebrew denominal verbs and the output-output correspondence. Phonology 16/3: 401-442.

\begin{abstract}
This article offers supporting evidence for the Root-and-Template model of morphology. It is argued that the consonantal root and the template are the basic morphological units handled in Tagnawt and Taqjmit (two secret languages used by women in Tashlhiyt Berber). Speakers are able to extract from Tashlhiyt forms only root consonants, and then disguise them by means of various morphological operations. In both languages, the root appears as fundamentally triliteral; bi- and monoconsonantals resorting to epenthesis (in Tagnawt) or incorporating affixes as root consonants (in Taqjmit) in order to achieve the required triliterality. As for templates, each of these secret languages displays a fixed-shape template over which certain morphological operations are realized, including gemination, reduplication and affixation. In addition, two major constraints are set, namely strict repetition in Taqjmit and template satisfaction in Tagnawt. These constraints prove necessary in explaining the size variations in Taqjmit forms, as well as the behaviour of certain quadriconsonantal forms in Tagnawt, where the medial vowel a is replaced with schwa [ə].
\end{abstract}

\title{
KeYwORDS
}

Morphophonology, consonantal root, templates, secret languages, Tashlhiyt Berber, Taqjmit, Tagnawt. 